\title{
Diseño de Neuromicrocurrículos para Carreras de Educación Superior
}

\section{Marcel Oswaldo Méndez Mantuano,}

Magister en Gestión Ambiental, Departamento de Investigación del Instituto

Tecnológico Superior Juan Bautista Aguirre, Ecuador

\section{Darwin Vicente Apolo Robles,}

Ingeniero Agroindustrial, Docente Investigador del Instituto Tecnológico

Superior Juan Bautista Aguirre, Ecuador

Rolando Gastón Viteri Gómez,

Licenciado en Ciencias de la Educación Especialidad Ingles, Coordinador de

la Carrera de Seguridad Ciudadana y Orden Público del Instituto

Tecnológico Superior Juan Bautista Aguirre, Ecuador

\section{Gladys Johanna Quijije Román,}

Licenciada en Ciencias de la Comunicación Social, Docente Investigadora del Instituto Tecnológico Superior Juan Bautista Aguirre, Ecuador

\section{Marlene Jacqueline Cortez Vargas,}

Licenciada en Ciencias de la Educación Mención Informática, Docente Investigadora del Instituto Tecnológico Superior Juan Bautista Aguirre,

Ecuador

Jennifer Lisenya Mantuano Flores,

Licenciada en Ciencias de la Educación mención Lengua Inglesa y

Lingüística, Docente investigadora de la Unidad Educativa Galo Plaza

Lasso, Ciudad de Daule, Ecuador

\section{Doi:10.19044/esj.2019.v15n19p22～URL:http://dx.doi.org/10.19044/esj.2019.v15n19p22}

\section{Resumen}

Aunque arriesgada y potencialmente blanda, la actual exploración enlaza a dos ciencias poco abordadas de forma integral como son las neurociencias y las ciencias de la educación, esto con el propósito de que la investigación sirva como referencia para diseñar la estructura fundamental de los componentes que debe poseer un neuromicrocurrículo. La propuesta presentada del diseño pedagógico hace énfasis en las emociones que se encuentran involucradas en los procesos normales de enseñanza y aprendizaje, para determinar cuáles deberían ser potenciadas por las metodologías educativas que se encuentran plasmadas en los microcurrículos. De igual forma se determina la necesidad de establecer contenidos curriculares que 
desarrollen los aspectos biológicos neuronales para enlazar los conocimientos con aspectos emocionales, de tal forma, que el aprendizaje dinamice la plasticidad cerebral para modelar conocimientos sustantivos en la memoria a largo plazo de los estudiantes. La investigación tiene un enfoque cualitativo, con un diseño documental y un alcance descriptivo.

Palabras claves: Cerebro, currículo, educación superior, emociones, microcurrículo, neurocurrículo, neuronal

\section{Design of Neuromicrocurriculum for Higher Education Careers}

\section{Marcel Oswaldo Méndez Mantuano,}

Magister en Gestión Ambiental, Departamento de Investigación del Instituto

Tecnológico Superior Juan Bautista Aguirre, Ecuador

Darwin Vicente Apolo Robles,

Ingeniero Agroindustrial, Docente Investigador del Instituto Tecnológico

Superior Juan Bautista Aguirre, Ecuador

Rolando Gastón Viteri Gómez,

Licenciado en Ciencias de la Educación Especialidad Ingles, Coordinador de

la Carrera de Seguridad Ciudadana y Orden Público del Instituto

Tecnológico Superior Juan Bautista Aguirre, Ecuador

Gladys Johanna Quijije Román,

Licenciada en Ciencias de la Comunicación Social, Docente Investigadora

del Instituto Tecnológico Superior Juan Bautista Aguirre, Ecuador

\section{Marlene Jacqueline Cortez Vargas,}

Licenciada en Ciencias de la Educación Mención Informática, Docente Investigadora del Instituto Tecnológico Superior Juan Bautista Aguirre,

Ecuador

Jennifer Lisenya Mantuano Flores,

Licenciada en Ciencias de la Educación mención Lengua Inglesa y Lingüística, Docente investigadora de la Unidad Educativa Galo Plaza

Lasso, Ciudad de Daule, Ecuador

Abstract

Although risky and potentially soft, the current exploration links two sciences that are not fully addressed, neurosciences and education sciences; 
with the purpose that the research serves as a reference to design the fundamental structure of the components that a neuromicrocurriculum must possess. The presented proposal of the pedagogical design, emphasizes the emotions that are involved in the normal processes of teaching and learning, to determine which should be enhanced by the educational methodologies that are embodied in the microcurricula. The need to establish curricular contents that develop the neurological biological aspects is determined, to link the knowledge with emotional aspects, in such a way that the learning dynamizes the cerebral plasticity to model substantive knowledge in the long term memory of the students. The research has a qualitative approach, with a documentary design and a descriptive scope.

Keywords: Brain, curriculum, higher education, emotions, microcurriculum, neurocurriculum, neuronal

\section{Introducción}

Para algunos, el cerebro humano es considerado el órgano más fascinante del universo, por ello sigue intrigando y sorprendiendo a los científicos, pues en este se estructuran las ideas y pensamientos de las personas. Esta masa encefálica posee un peso aproximado de $1,4 \mathrm{~kg}$ (en un adulto promedio), con un volumen de $1.400 \mathrm{~cm}^{3}$ y 100.000 millones de neuronas, que en promedio tienen unas 10.000 sinapsis. La neurona es la unidad anatómica y funcional cerebral, siendo esta célula la encargada de la recepción, integración y transmisión de señales químicas. Dichas células especializadas forman redes neuronales muy complejas, las cuales sirven de comunicación entre ellas, comunicación que es denominada comúnmente como sinapsis. Las señales que llegan a las neuronas suelen ser químicas, y las dendritas las convierten en señales eléctricas, que son incorporadas al resto de señales procedentes de otras dendritas; si dentro de una neurona la señal es importante, el potencial eléctrico viaja por el axón hacia la sinapsis (Valdés, 2018).

Desde hace dos décadas varias ciencias profundizan el estudio del sistema nervioso central humano para entender los aspectos estructurales y funcionales que se desarrollan en el mismo, ayudados por tecnología de punta como las resonancias magnéticas, las neuroimágenes, entre otras; las cuales han permitido visualizar las interacciones motoras que intervienen en el lenguaje, la memoria y la atención. Estas investigaciones revelaron que el desarrollo cerebral inicia desde las primeras etapas embrionarias, las cuales se mantienen a lo largo de la vida de las personas, y que son afectadas por factores genéticos, sociales, culturales, educativos que definen al sujeto (Campos, 2010). 
Las neurociencias han experimentado notables avances en la actualidad, dado el desarrollo tecnológico de técnicas que permiten mapear al cerebro humano en sus actividades cognitivas. La mayoría de los estudios se han enfocado en el análisis de la memoria y el aprendizaje, lo que ha permitido conocer cómo este órgano codifica la información que recibe del mundo exterior, y además se ha tratado de interpretar cómo los procesos de aprendizaje se llevan a cabo en el mismo. Estos avances están siendo aprovechados por ciencias sociales como la educación, las cuales intentan diseñar un marco teórico y científico que permita mejorar los aspectos pedagógicos, ya que es sabido que las bases biológicas y cognitivas forman las bases de la enseñanza y el aprendizaje de las personas, y el conocimiento de estas "leyes", permitirán diseñar currículos asimilables para el cerebro (Caballo \& Portero, 2018).

En la actualidad la palabra "neuro" agrupa diferentes ciencias, desde ramas biológicas que abordan los aspectos médicos-neurológicos, hasta las sociales que interpretan la psiquis a través de los actos. Con los aportes de las neurociencias, la educación ha reorientado sus objetivos, ya que en la actualidad se desea comprender la integralidad de los estudiantes como personas desde los cimientos biológicos involucrados en la construcción de sus pensamientos. De la fusión de los conceptos de neurología y educación emergió la neurodidáctica (término acuñado por Gerhard Friedrich y Gerhard Preiss en 1988), cuyo propósito es entender la forma de mejorar los procesos de aprendizaje para potenciar el desarrollo cognitivo cerebral en diferentes áreas. Se está tratando de promover la unión de la educación, la biología y la psicología para desarrollar una nueva área científica que aborde las causales de la mente, el cerebro y la educación, ya que si en el cerebro es donde se localiza el aprendizaje, no es lógico excluirlo de los procesos de enseñanza (Politécnico Superior de Colombia, 2017).

Para las neurociencias el aprendizaje es una variación en las conexiones sinápticas que producen el pensamiento y el comportamiento, las cuales derivan de la información del exterior (interacciones o experiencias de la vida). La información sensorial es recibida por varias zonas del encéfalo, y esta es transmitidas a otras áreas especializadas donde es procesada la información de las células, para que posteriormente sean proyectadas en forma de estímulos sensoriales. Estudios han determinado que el encéfalo construye imágenes de lo percibido del mundo físico a través de un proceso de integración selectiva de la información de las aferencias sensoriales, las cuales se encuentran conectadas de una manera ordenada. También se ha destacado que las actividades relativas al aprendizaje son capaces de modificar las conexiones neuronales cerebrales, siempre que los estímulos externos ayuden a este fin (Valdés, 2018). 
Actualmente las neurociencias y la educación no han alcanzado integrarse de manera efectiva, ya que no existen consensos que logren articular eficientemente los conocimientos generados en estos saberes, dadas las posturas divergentes entre defensores y detractores. Las críticas señalan que la extrapolación de los resultados obtenidos en seres humanos y animales no es generalizable a los contextos áulicos, ya que los procesos educativos tienen un cariz social y en un bajo porcentaje biológico; razón por la cual el ambiente, el entorno, el bienestar, la creatividad, la salud, tienen mayor impacto educativo. Por otro lado, quienes señalan las ventajas de la integración de estas dos ramas, afirman que los principios, mecanismos y teorías de la educación pueden ser ampliados y corregidos si estas se analizan a través de las neurociencias cognitivas; adicionalmente los principios de las neurociencias pueden converger en investigaciones educativas interdisciplinarias (Barrios, 2016).

Desde hace mucho tiempo se mantuvo la idea de que el estudio del cerebro pertenecía únicamente a los psicólogos y neurólogos, lo cual fue descartado de manera apropiada en los últimos años (Ortiz, 2012). En el ámbito educativo, se consentía que el profesor poco se beneficiaba de conocer el funcionamiento de la mente; sin embargo, los nuevos estudios demuestran que existen áreas cerebrales específicas que se desarrollan paulatinamente de acuerdo al desarrollo físico de las personas, y el conocimiento de esto ayudaría a desarrollar técnicas y estrategias que estimulen al cerebro de acuerdo a condiciones particulares de los grupos humanos para lograr un efectivo proceso de enseñanza-aprendizaje (De Zubiría, 2009).

Las neurociencias pretenden concebir las bases neuronales biológicas que desarrollamos en el interior del cráneo, mientras que la educación estructura las formas de aprendizajes coherentes para el cerebro. Al interrelacionarse ambas, es posible utilizar este conocimiento para informar y modificar los modelos cognitivos diseñados por las teorías de aprendizaje y con ello aportar en la interpretación del comportamiento humano (Gruart, 2014).

Es posible entonces definir a la neurodidáctica como "la disciplina científica que estudia la optimización y potenciación del aprendizaje basado en el desarrollo de todo el potencial del cerebro humano, analizando el pensamiento, las emociones y la inteligencia", siendo estos factores analizados desde la perspectiva neurológica y no solamente como el producto de procesos afectivos-cognitivos. La nueva propuesta educativa consiste en el diseño de la pedagogía del cerebro, o neuropedagogía y neurodidáctica, cuyo propósito es configurar y estimular las redes neuronales del cerebro para mejorar el desempeño de los estudiantes y permitir la interiorización eficiente de los conocimientos, y cuyos fundamentos serán las estrategias pedagógicas, didácticas, curriculares y evaluativas (Ortiz, 2015). 
En la educación existen neuromitos (ideas falsas sobre el funcionamiento del cerebro) que, lejos de ayudar a los procesos de enseñanza, son un serio riesgo para los mismos. El desconocimiento en las áreas de las neurociencias ha proliferado con rapidez estas aseveraciones erróneas del comportamiento cerebral, y en algunos casos se han afianzado de manera robusta, pero perjudicial (Acevedo, 2016). Entre los neuromitos difundidos con mayor frecuencia están: sólo usamos el $10 \%$ de nuestro cerebro, las personas usan preferiblemente uno de los hemisferios del cerebro (derecho o izquierdo), existen múltiples inteligencias que utilizan áreas diferentes del cerebro, existen estilos dominantes de aprendizaje y al enseñar la modalidad sensorial dominante esta permitirá mejores resultados educativos, beber mucha agua en la infancia ayuda a mantener un cerebro mejor hidratado (Rodrigues, Abreu, \& Castro-Caldas, 2013).

Por otro lado, cada clase es un territorio de diversidades, ya que cada persona tiene sus propias motivaciones, experiencias, intereses, capacidades y, de manera general, una forma única de ver la escuela, el aula y la dinámica de integrarse al aprendizaje. Los currículos oficiales prescriben y habilitan los contenidos y metodologías que se integrarán en la interrelación de docentes y estudiantes, donde los primeros realizan una planificación estratégica para cumplir con los objetivos propuestos. Entonces, se traduce que el sentido de la enseñanza es el cumplimiento de los objetivos de aprendizaje por parte de los estudiantes en los tiempos establecidos, donde se parte de la premisa "que todos somos capaces y todos podemos aprender", razón por la cual se deben repensar las configuraciones didácticas; es decir, se deben reconstruir de manera consensuada y periódica los contenidos académicos que se imparten, de tal forma que sean consideradas las particularidades evolutivas de la sociedad (Ferreyra, Sandrome, \& Vidales, 2017).

Aquí es donde toma relevancia el diseño de los neurocurrículos, los cuales deberían integrar las características del cerebro (demostradas por las neurociencias) en la forma de cómo este órgano asimila la información del mundo exterior, ya que una adecuada configuración didáctica permite enseñar y favorecer la comprensión de todos los estudiantes; es decir, consentir que ellos individualmente formen los procesos de construcción y reconstrucción de los conocimientos (Litwin, 2000).

A estos procesos automáticos de construcción, las neurociencias los denominan "plasticidad cerebral", el cual es un proceso de adaptación constante donde las neuronas consiguen aumentar las conexiones con otras neuronas de forma estable, a causa de las experiencias, el aprendizaje, la estimulación sensorial y cognitiva (Aguilar, Caballero, Ormea, Salazar, Loayza, \& Muñoz, 2017).

Para articular lo anteriormente mencionado, es necesario el diseño de neurocurrículos basados en el funcionamiento del cerebro, añadiendo 
concepciones básicas de los currículos tradicionales. Entiéndase que el currículo es la inserción armónica de las ciencias "Pedagogía" y "Didáctica", en las que se relacionan los aspectos educativos tales como: problema, objeto, objetivo, contenido, método, resultado, evaluación, entre otros. Por ello, se identifica que el currículo es la concepción didáctica de teorías, principios, categorías, regularidades, en un objeto particular de aprendizaje (Ortiz, 2010).

En este sentido, la presente investigación plantea diseñar la estructura fundamental para la creación de neuromicrocurrículos que sean aplicables en las aulas de las instituciones de educación superior, sin descartar que estas premisas sirvan como referencias para los otros niveles de estudios. Para cumplir con este objetivo inicialmente se plantea conocer los procesos básicos del cerebro que están involucrados en el aprendizaje, así como la descripción de las principales hormonas que desencadenan las principales emociones en las personas; posteriormente se indaga en la estructura de los diseños curriculares vigentes para los estudiantes de educación superior en el Ecuador, y finalmente se diseña una propuesta esquemática para la estructuración de neuromicrocurrículos.

\section{Metodología}

La presente investigación tiene un enfoque cualitativo, ya que se exploran postulados científicos en el área neuronal y educativo para integrarlos de manera conjunta y desarrollar una propuesta para la elaboración de neuromicrocurrículos en las carreras de educación superior del Ecuador.

Tiene un diseño de tipo documental, dado que la información abordada proviene de material suplementario, tales como tesis y revistas científicas, las cuales han sido filtradas de manera exhaustiva para que las pesquisas presentadas tenga un fuerte respaldo académico e investigativo. La información recolectada permitió conocer las emociones y hormonas que están involucradas en el aprendizaje; además se analizaron las partes que integran un currículo y finalmente se consideró la "Guía Metodológica de Presentación y Aprobación de Proyectos de Carrera" elaborada por el Consejo de Educación Superior (CES) en el año 2016, para perfilar la propuesta de la estructura neuromicrocurrícular.

La investigación tiene un alcance descriptivo, ya que se explicó el funcionamiento de los principales neurotransmisores que están relacionados de manera directa con el aprendizaje, lo cual permitió el enlazamiento de dos ramas científicas muy poco estudiadas en la actualidad (de forma integral). 


\section{Desarrollo}

\section{Procesos cerebrales involucrados en el aprendizaje}

Las respuestas de los organismos está determinada por los estímulos del medio que lo sitia y son medidas por dos sistemas diferentes ubicados en el diencéfalo, de manera preferencial en el hipotálamo (Jung, 1992).

La comunicación interneuronal (también denominada sinapsis cerebral), en conjunto con las múltiples redes que forman estas neuronas, estimulan al cerebro y diseñan la estructura del gran procesador central del cuerpo humano. Todas estas interrelaciones crean los fundamentos del aprendizaje y permiten observar la plasticidad neuronal, la cual se interpreta como la capacidad que posee el sistema nervioso de permitir las uniones neuronales y por lo tanto la eficiencia sináptica (de célula a célula y de manera agrupada), la misma que deriva de los estímulos que percibe nuestro cerebro. El discernimiento de la plasticidad cerebral ayudará en los procesos de aprendizaje. Esta característica es posible visualizarla cuando existen cambios en la conducta adquirida de las personas, ya que la adherencia de nuevas experiencias reemplaza a las anteriores; es decir, el cerebro se encuentra en constante aprendizaje y desaprendizaje, modificando la sinapsis de forma anatómica y funcional. En otras palabras, para que acontezca un eficaz proceso de aprendizaje, deberá existir la mayor cantidad de conexiones neuronales presentes en nuestros cerebros (Velásquez, Remolina, \& Calle, 2009).

También las emociones desempeñan un papel preponderante en el aprendizaje, ya que estas desencadenan los cambios químicos que modifican los estados anímicos, de conducta e incluso la forma en que percibimos la vida. Las emociones son las precursoras para la toma de decisiones (ya sean superficiales o transcendentales); todo lo que percibimos del exterior es interiorizado y proyectado en forma de sentimientos, tales como la calma, la ira, el dolor, el placer, etc. La corteza prefrontal es la zona que permite a la memoria activa y a los recuerdos expresarse en el cerebro. Las emociones encasilladas como negativas (tristeza, depresión, estrés, entre otras), son capaces de interrumpir de manera significativa la capacidad cognitiva de la mente (memoria activa); es decir, una persona sometida a tales emociones, no será capaz de retener información relevante de una actividad por un lapso largo de tiempo (Velásquez, Remolina, \& Calle, 2009).

Existen dos formas para identificar las emociones humanas, la primera consiste en la medición de las variables o dimensiones del sistema afectivo, y la segunda reconoce e interpreta las emociones proyectadas a través de los gestos faciales considerados como universales. El sistema emotivo es analizado como un arreglo circular (también denominado rueda de la emoción), en el que sobresalen los ejes del agrado, el desagrado, la excitación y la relajación, y donde cada uno de ellos agrupa las diferentes emociones que experimentamos, entre los que encontramos: la altivez, el valor, la alegría, la 
satisfacción, el entusiasmo, la diversión, el vigor, la tensión, la duda, la ira, el odio, la aversión, el dolor, el desagrado, la humillación, el miedo, la tristeza, la frustración, la apatía, el aburrimiento, el agotamiento, la calma, la certeza, la compasión, el amor, el deseo, el placer, entre otros (Díaz \& Flores, 2001).

Según el diccionario de neurociencia, las emociones son reacciones conductuales y subjetivas que son producidas por la información del mundo exterior e interior (memoria), que vienen acompañadas de procesos neurovegetativos donde el sistema límbico es uno de los principales responsables en la generación de las conductas emocionales (Mora \& Sanguinetti, 2004).

Según Mora (2008), los siete pilares básicos de las emociones son:

1. Las emociones actúan como un sistema defensivo frente a los estímulos negativos, y nos acercan a los estímulos que son placenteros para nuestra biología (beber agua, jugar, etc.). En otras palabras, las emociones nos motivan a conseguir o evitar algo que sea beneficioso o dañino para las personas.

2. Las emociones permiten la flexibilidad de la conducta (respuesta) frente a un estímulo; es decir, no existen reacciones mecánicas, sino que el cerebro evalúa el evento y diseña la acción apropiada al mismo, de acuerdo a las circunstancias presentes.

3. Las emociones sirven para activar de manera instantánea múltiples áreas del cerebro y del cuerpo.

4. Las emociones mantienen la curiosidad y el interés por lo nuevo (alimentos, aprendizajes, etc.).

5. Las emociones sirven como lenguaje universal de las personas, mediante el mecanismo de la empatía.

6. Las emociones permiten recrear y almacenar en la memoria recuerdos asociados a episodios emocionales (positivos y negativos).

7. Las emociones juegan un rol preponderante en el razonamiento (en las ideas clasificadas como buenas o malas), y por ende en la toma de decisiones voluntarias.

Autores como Mendoza (2017) limitan a 5 las emociones básicas, las mismas que involucran diferentes hormonas y presentan las siguientes características:

\begin{tabular}{|l|l|c|}
\hline Emoción & Característica & $\begin{array}{c}\text { Hormona } \\
\text { involucrada }\end{array}$ \\
\hline \multirow{2}{*}{ Alegría } & $\begin{array}{l}\text { La alegría la produce un neurotransmisor } \\
\text { denominado dopamina, el cual tiene la } \\
\text { función de suministrar los sentimientos de } \\
\text { refuerzo y compasión, permitiéndonos } \\
\text { tener un buen estado de ánimo. Por otro } \\
\text { lado, los bajos niveles de esta hormona nos } \\
\text { hacen disminuir de manera considerable los }\end{array}$ & $\begin{array}{c}\text { Dopamina } \\
\left(\mathrm{C}_{8} \mathrm{H}_{11} \mathrm{NO}_{2}\right)\end{array}$ \\
\hline
\end{tabular}




\begin{tabular}{|c|c|c|}
\hline & $\begin{array}{l}\text { aspectos sociales. También es considerada } \\
\text { como una neurohormona ; es decir, que la } \\
\text { podemos encontrar en la sinapsis neuronal } \\
\text { producida por el hipotálamo. }\end{array}$ & \\
\hline Amor & $\begin{array}{l}\text { El amor es producido cuando se unen dos } \\
\text { hormonas, la dopamina y la oxitocina; esta } \\
\text { última se produce en la hipófisis (conocida } \\
\text { como la molécula de amor). Es considerada } \\
\text { como un neurotransmisor y en bajas } \\
\text { concentraciones inhibe las capacidades de } \\
\text { relacionarse con otras personas. Tiene una } \\
\text { función directa en los aspectos sociales, de } \\
\text { placer, el orgasmo, la generosidad, la } \\
\text { confianza, entre otros. }\end{array}$ & $\begin{array}{c}\text { Oxitocina } \\
\left(\mathrm{C}_{43}-\mathrm{H}_{66}-\mathrm{N}_{12}-\mathrm{S}_{2}\right) \\
\text { Dopamina }\end{array}$ \\
\hline Odio & $\begin{array}{l}\text { La serotonina es un neurotransmisor muy } \\
\text { relacionado con el control de las emociones } \\
\text { y el estado de ánimo. Esta hormona } \\
\text { funciona como inhibidor del enfado, de la } \\
\text { temperatura del cuerpo, de la ira, de la } \\
\text { agresión, controla las actividades motoras, } \\
\text { regula los ritmos cardíacos y del sueño, } \\
\text { mantiene la estructura ósea, induce a la } \\
\text { división celular, regula el deseo sexual. Los } \\
\text { bajos niveles de serotonina ocasionan } \\
\text { agresividad. }\end{array}$ & $\begin{array}{c}\text { Serotonina } \\
\left(\mathrm{C}_{10} \mathrm{H}_{12} \mathrm{~N}_{2} \mathrm{O}\right)\end{array}$ \\
\hline Tristeza & $\begin{array}{l}\text { La tristeza, la falta de atención, la poca } \\
\text { concentración, la depresión y el estrés son } \\
\text { ocasionados por los bajos niveles del } \\
\text { neurotransmisor llamado noradrenalina o } \\
\text { norepinefrina, el cual cumple la función de } \\
\text { mantenernos alerta; en otras palabras, } \\
\text { activa nuestros instintos primarios o } \\
\text { naturales relacionados a la atención. Esta } \\
\text { hormona tiene un efecto sobre el estado } \\
\text { motivacional y energético, participando } \\
\text { activamente junto a la dopamina en la } \\
\text { regulación del aprendizaje, memoria y } \\
\text { sensación de recompensa. }\end{array}$ & $\begin{array}{l}\text { Noradrenalina } \\
\left(\mathrm{C}_{8} \mathrm{H}_{11} \mathrm{NO}_{3}\right)\end{array}$ \\
\hline Miedo & $\begin{array}{l}\text { El miedo está relacionado con la } \\
\text { interrelación de la dopamina, la serotonina } \\
\text { y la noradrenalina, las cuales causan } \\
\text { diferentes reacciones en nuestro cuerpo, por } \\
\text { ejemplo: aceleración de la función } \\
\text { pulmonar y cardíaca, inhibición del sistema } \\
\text { digestivo, dilatación de las pupilas, etc. }\end{array}$ & $\begin{array}{l}\text { Dopamina, } \\
\text { Serotonina, } \\
\text { Noradrenalina }\end{array}$ \\
\hline
\end{tabular}

Tabla 1. Características de los neurotransmisores que producen la alegría, el amor, el odio, la tristeza y el miedo 


\section{Estructura de los diseños curriculares}

Los currículos pedagógicos de educación superior siguen un criterio similar a los de sus pares curriculares de los niveles previos, donde la diametral diferencia entre estos radica en que las competencias que adquiere el estudiante (universitario o técnico) le permitirán acceder a los conocimientos, destrezas y actitudes para ejecutar actividades laborales (profesionales) de manera autónoma, creativa, colaborativa, analítica y organizativa en su futuro entorno de trabajo.

El término currículo ha tenido varias interpretaciones por parte de diferentes autores, siendo entonces un término polisémico; sin embargo, existe una convergencia conceptual, pudiéndose interpretar como una planificación estratégica (educativa) de manera anticipada y con objetivos específicos claros y medibles. En la actualidad, el término se refiere a la simbiosis de las estrategias educativas, los contenidos, los objetivos de aprendizaje, las experiencias educativas, el entorno, la evaluación, estilos de aprendizaje, la programación y resultados de aprendizaje (Cargua, 2005).

El currículo posee dos dimensiones, la dimensión estática o también denominado macrocurrículo (nivel más alto) y mesocurrículo (nivel medio o nexo entre el macro y micro currículo), y la dimensión dinámica señalado como microcurrículo (nivel más bajo). Todos ellos estan interrelacionados entre sí como un sistema integrado, inseparables en la práctica educativa, donde cada uno de ellos se materializa en varios documentos curriculares con exigencias y requisitos propios (Ocaña, 2009).

Para entender a plenitud el concepto de microcurrículo es necesario partir de la noción dada a currículo, ya que este último abarca de manera integral al primero, no existiendo uno sin el otro (Urbina \& Agudelo, 2016). Aunque existen diferentes componentes para la estructuración del currículo (macro, meso y micro currículo), se tomará en consideración la estructura del diseño curricular dada por el Consejo de Educación Superior (CES) del 2016, ya que la misma se encuentra en vigencia para las carreras de Educación Superior del Ecuador, y la cual menciona lo siguiente para cada uno de los componentes curriculares:

1. El macrocurrículo aborda aspectos tales como, el análisis de la pertinencia de una determinada carrera (en un lugar geográfico). Dentro del mismo se detallan las tendencias y tensiones del campo de formación profesional que se desea ofertar, el cual es una proyección analítica del futuro de dicho programa y de sus teóricos efectos en áreas científicas y académicas. Como soporte se deben detallar los cuerpos legales que rigen a la educación (nivel superior), con especial énfasis en aquellos estatutos o reglamentos que justifiquen el programa de estudios. De igual manera es necesario conocer el mercado laboral y de empleabilidad de los futuros graduados, por lo que se realizan encuestas dirigidas a los entes académicos y productivos de la zona 
para conocer la demanda actual y futura de profesionales en la rama diseñada. También es necesario determinar de manera directa las potenciales problemáticas que afrontará la carrera, realizando un diagnóstico de la situación actual a través de un análisis de la demanda de los estudiantes en niveles previos de formación. Finalmente, la valoración de la brecha laboral (oferta - demanda) esclarecerá si existen espacios para los futuros profesionales en un determinado lapso de tiempo, permitiendo la justificación de la creación de la carrera (Méndez, Campi, Huayamave \& Lozada, 2017).

2. $\quad$ En el mesocurrículo se detallan los objetivos de la carrera, los cuales deben guardar relación con los resultados de aprendizaje; por su parte, los objetivos específicos deberán tener relación directa con la pertinencia y los objetivos de aprendizaje. Posteriormente se especifican las políticas de permanencia, promoción estudiantil y de la dinámica para fomentar la diversidad en todas sus formas e impulsar el aprendizaje intercultural. Dentro de este apartado se establece la malla curricular, el número de horas por asignatura, el flujograma, las unidades de organización curricular de las asignaturas (básicas, profesionales o de titulación) y los campos de formación (teóricos; adaptación e innovación tecnológica; comunicación y lenguaje; e integración de saberes, contextos y cultura). También se detallan las estrategias para incorporar la investigación en los procesos de enseñanzaaprendizaje (Méndez, Campi, Huayamave \& Lozada, 2017).

3. La estructura microcurricular está delineada por los siguientes descriptores: nombre de la asignatura, campo de formación (teóricos; adaptación e innovación tecnológica; comunicación y lenguaje; e integración de saberes, contextos y cultura), unidad de organización curricular (básicas, profesionales o de titulación), número de período académico (nivel de estudio o semestre), número total de horas de las asignatura (de acuerdo a la malla curricular), organización de aprendizajes por modalidad o número de horas destinadas a cada componente (docencia, prácticas de aprendizaje, aprendizaje autónomo), objetivos de la asignatura, resultados de aprendizaje, contenidos mínimos de la asignatura (detalle de las unidades de estudio), estrategias metodológicas y recursos didácticos (Consejo de Educación Superior, 2016).

De manera aclaratoria se puede indicar que el currículo es el conjunto de criterios sociales, académicos, empresariales, legales y productivos que en primera instancia justifican la creación y diseño de una carrera. Posteriormente se detallan los planes de estudio, programas generales, procesos, metodologías y las políticas en los cuales se fundamentará; y finalmente se detallan los contenidos educativos que deben analizar las diferentes asignaturas en los diferentes niveles de estudios. Esta integralidad se define como currículo (en la actualidad).

Los microcurrículos son una porción específica del currículo, los cuales reflejan los valores institucionales de formación, su enfoque, las bases 
conceptuales que estructuran las metodologías, para alcanzar el perfil de egreso y los objetivos de aprendizaje propuestos.

La estructura microcurricular para asignaturas en carreras de educación superior en la cual se basa esta investigación es la siguiente:

\begin{tabular}{|c|c|}
\hline \multicolumn{2}{|l|}{ Datos generales de la asignatura } \\
\hline Nombre de la asignatura: & $\begin{array}{l}\text { Nombre de la asignatura dentro de un determinado } \\
\text { nivel de estudios, período académico o semestre. }\end{array}$ \\
\hline Campo de formación: & $\begin{array}{l}\text { Se clasifican en: Fundamentos teóricos; Adaptación e } \\
\text { innovación tecnológica; Comunicación y lenguaje; e } \\
\text { Integración de saberes, contextos y cultura. }\end{array}$ \\
\hline $\begin{array}{l}\text { Unidad de Organización } \\
\text { curricular: }\end{array}$ & $\begin{array}{l}\text { Se clasifican en: Básicas, Profesionales y de } \\
\text { Titulación. }\end{array}$ \\
\hline Número de período académico: & $\begin{array}{l}\text { También llamado nivel de estudios, período } \\
\text { académico o semestre. }\end{array}$ \\
\hline Número de horas de la asignatura: & Están determinadas por la malla curricular. \\
\hline $\begin{array}{l}\text { Número de horas por cada } \\
\text { componente: }\end{array}$ & $\begin{array}{l}\text { Se desglosan las horas de las asignatura en los } \\
\text { componentes de docencia (dentro del aula), prácticas } \\
\text { (talleres, laboratorios, simuladores, etc.) y trabajo } \\
\text { autónomo (tareas asignadas para fomentar en el } \\
\text { estudiante el autoaprendizaje autorregulado e } \\
\text { independiente). }\end{array}$ \\
\hline Objetivos generales y específicos: & $\begin{array}{l}\text { El objetivo general debe responder al "qué, cómo y } \\
\text { para qué" en función de los resultados de aprendizaje. } \\
\text { Por su parte, los objetivos específicos deben estar } \\
\text { relacionados con el conocimiento, la pertinencia, los } \\
\text { aprendizajes y deben aportar a la consecución del } \\
\text { objetivo general. }\end{array}$ \\
\hline Resultados de aprendizaje: & $\begin{array}{l}\text { Es lo que se espera que el estudiante deba saber, } \\
\text { comprender y/o sea capaz de demostrar una vez } \\
\text { terminadas las unidades propuestas. }\end{array}$ \\
\hline $\begin{array}{l}\text { Descripción de contenidos } \\
\text { curriculares: }\end{array}$ & $\begin{array}{l}\text { Es la unidad básica del aprendizaje, en donde se } \\
\text { establecen las unidades curriculares que abordará la } \\
\text { asignatura, detallando los sub capítulos (o temas) por } \\
\text { cada unidad. La organización sigue criterios lógicos, } \\
\text { como por ejemplo, se inician en las nociones } \\
\text { fundamentales (básicas), hasta las complejas } \\
\text { (estructuradas). }\end{array}$ \\
\hline Recursos didácticos: & $\begin{array}{l}\text { Son aquellos materiales didácticos o educativos que } \\
\text { sirven como mediadores para el desarrollo y } \\
\text { enriquecimiento del alumno y facilitan la } \\
\text { interpretación de los contenidos. }\end{array}$ \\
\hline Recomendaciones metodológicas: & $\begin{array}{l}\text { Son los pasos estructurados que organizan los } \\
\text { aprendizajes, de tal manera que se logran los } \\
\text { objetivos propuestos. Estas metodologías están } \\
\text { fundamentadas en investigaciones teóricas o prácticas } \\
\text { que garantizan la forma en la cual se impartirán los } \\
\text { conocimientos. }\end{array}$ \\
\hline
\end{tabular}




\begin{tabular}{|l|l|}
\hline Consultas de referencias: & $\begin{array}{l}\text { Son los documentos (libros, revistas científicas, } \\
\text { ensayos, tesis, etc.) a los cuales un estudiante puede } \\
\text { acudir para profundizar los conocimientos. En lo } \\
\text { posible deben estar actualizados o con vigencia } \\
\text { científica. }\end{array}$ \\
\hline
\end{tabular}

Tabla 2. Estructura microcurricular estandar. Fuente: CES (2016)

\section{Diseño del neuromicurrículo}

La fusión de los diseños microcurriculares (basado en el diseño por competencias), con la comprensión del funcionamiento básico del cerebro, está determinada por la siguiente simbiosis hipotética:

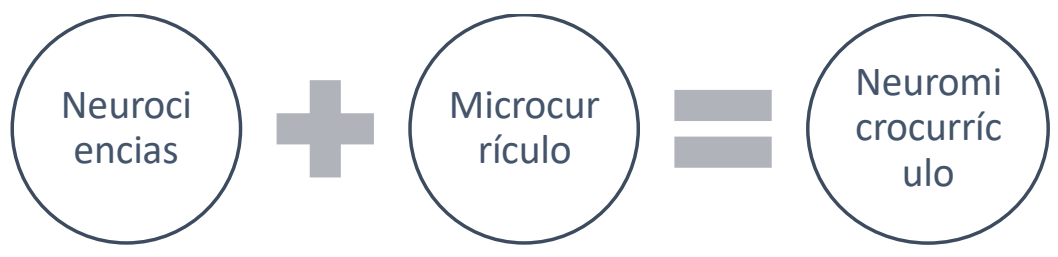

Es necesario comprender los objetivos que persiguen los neuromicrocurrículos, ya que el pleno conocimiento de esto permitirá estimular de manera positiva el aprendizaje y evitar (en lo posible) la disgregación de los fines de los neuromicrocurrículos. Entre los objetivos proyectados están los siguientes:

1. Conocer los neurotransmisores involucrados en el proceso de aprendizaje de los estudiantes de nivel superior.

2. Articular los campos de formación, los objetivos de la asignatura, los resultados de aprendizaje, los contenidos curriculares, los recursos didácticos y las metodologías de enseñanza de tal forma que se comprendan los procesos mentales efectuados durante el desarrollo del neuromicrocurrículo.

3. Estudiar cuáles son los recursos didácticos apropiados para el desarrollo de los neurocurrículos en estudiantes de nivel superior.

4. Analizar cuáles son las mejores metodologías de enseñanza que permitan ejecutar los neuromicrocurrículos en el aula.

5. Diseñar actividades pedagógicas que estimulen la producción natural de los neurotransmisores involucrados en los procesos de aprendizaje.

6. Evaluar de manera periódica los resultados alcanzados por la aplicación de los neurocurrículos en el aula.

7. Analizar los resultados alcanzados para diseñar y rediseñar de manera fundamentada las áreas de neuroeducación y neurodidáctica.

También es imperioso detallar qué se admite conceptualmente como microcurrículo, ya que en las secciones anteriores se especificó la estructura general del mismo (en Ecuador), sin abordar los postulados teóricos que debe seguir este segmento curricular. 
Para Durant y Naveda (2012), los microcurrículos por competencias son diseñados para analizar una unidad específica y/o área del conocimiento del plan de estudios determinado; por su parte Tejada y Tobon (2006), sostienen que los mismos pretenden orientar el aprendizaje, la docencia y la evaluación mediante la incorporación de los escenarios prácticos que garanticen la cohesión con los aspectos teóricos, los cuales se encontrarán respaldados por proyectos áulicos, aprendizaje basado en problemas, uso de mapas conceptuales, simulaciones y pasantías.

El objetivo microcurricular es orientar al estudiante y docente durante todo el proceso de aprendizaje. Igualmente permite el desarrollo oportuno de la planificación áulica, de tal forma que se impide la improvisación. También consiente la visualización de las necesidades de los estudiantes desde diferentes perspectivas, pudiéndose adaptar los contenidos a la evolución de los aprendizajes (Martínez \& Petit, 2013).

Con estos antecedentes, podemos articular los aspectos que comprenden los neuromicrocurrículos de la siguiente forma:

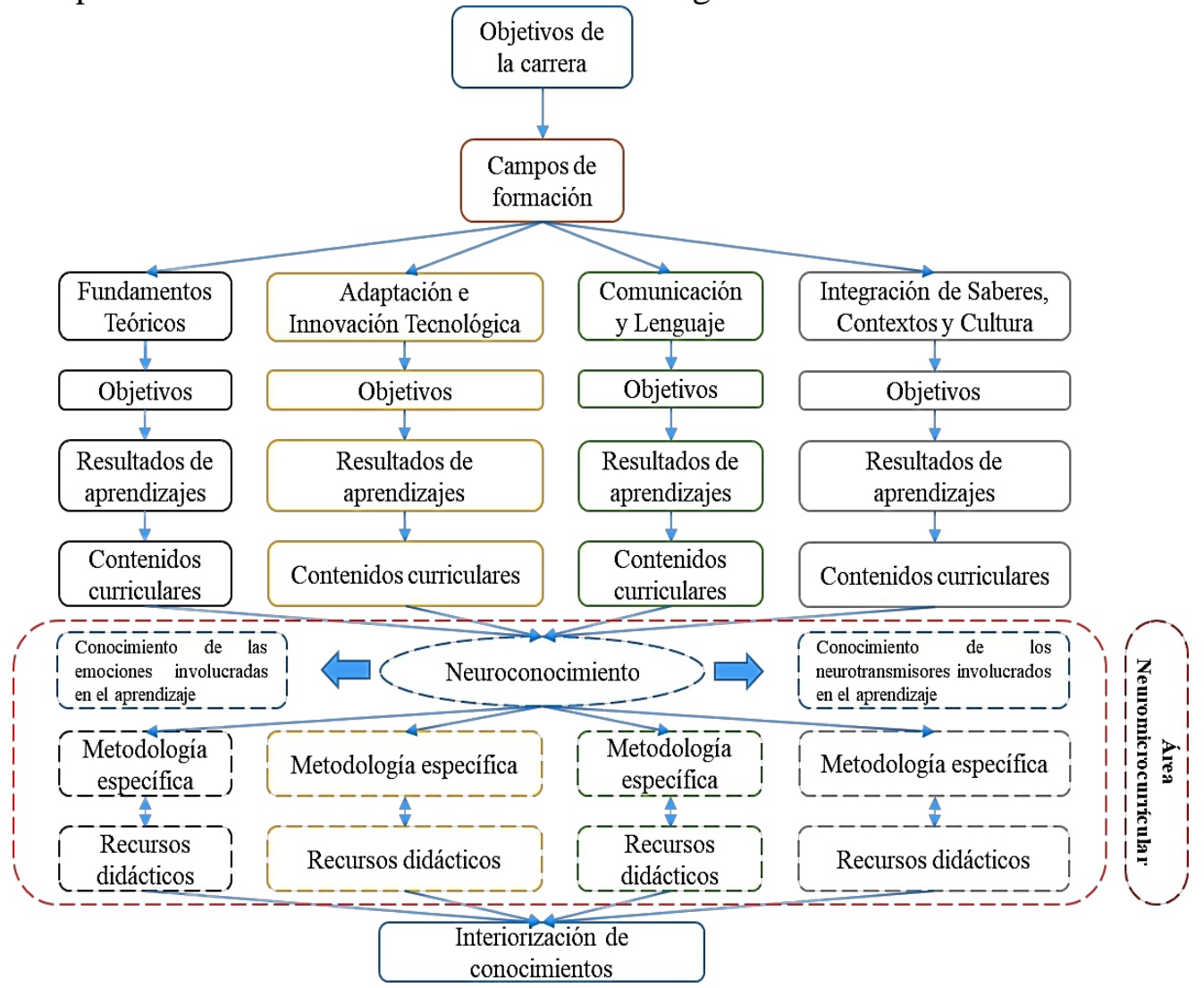

Figura 1. Especificación del área de competencia de los neurocurrículos. Fuente: Autores 
En base a la figura anterior, los fundamentos sobre los cuales giran los neuromicrocurrículos (de manera preponderante) se encuentran en la metodología de enseñanza y los recursos didácticos aplicados para la misma. Esto no quiere decir que los otros componentes no formen parte del diseño neurocurricular, sino que los mismos son las directrices referenciales para delinear las formas de enseñanza con sus respectivos materiales. En otras palabras, los neuromicrocurrículos son las estrategias puestas en práctica de los contenidos curriculares, con el objetivo de desarrollar los aspectos biológicos neuronales de los estudiantes.

Los neuromicrocurrículos no ayudan en la estructuración de las unidades de estudio, ya que el origen de los contenidos curriculares deriva de áreas superiores a las abordadas en los neuromicrocurrículos; es decir, los campos de formación estructuran a los objetivos de las asignaturas y estos a su vez modulan los resultados de aprendizajes.

A continuación se presenta la estructura general para el diseño de neuromicrocurrículos en los componentes metodológicos y de recursos didácticos:

\begin{tabular}{|c|c|c|c|c|}
\hline Componentes & Detalle & $\begin{array}{l}\text { Emociones } \\
\text { involucradas }\end{array}$ & $\begin{array}{l}\text { Neuro- } \\
\text { actividades }\end{array}$ & $\begin{array}{l}\text { Recursos } \\
\text { didácticos }\end{array}$ \\
\hline $\begin{array}{l}\text { Recomendacion } \\
\text { es } \\
\text { metodológicas }\end{array}$ & $\begin{array}{l}\text { Se debe especificar } \\
\text { la metodología } \\
\text { educativa que } \\
\text { permita a los } \\
\text { estudiantes de nivel } \\
\text { superior interiorizar } \\
\text { la información } \\
\text { analizada en cada } \\
\text { una de las unidades } \\
\text { curriculares. }\end{array}$ & $\begin{array}{l}\text { Se deben } \\
\text { promover } \\
\text { aquellas } \\
\text { emociones que } \\
\text { produzcan } \\
\text { altos niveles } \\
\text { dopamina, } \\
\text { oxitocina, } \\
\text { noradrenalina. }\end{array}$ & $\begin{array}{l}\text { Se deben } \\
\text { estudiar } \\
\text { aquellas } \\
\text { actividades } \\
\text { que permitan } \\
\text { la producción } \\
\text { natural de } \\
\text { neurotransmis } \\
\text { ores asociados } \\
\text { a las } \\
\text { emociones } \\
\text { deseadas. }\end{array}$ & $\begin{array}{l}\text { Se deben } \\
\text { analizar } \\
\text { aquellos } \\
\text { recursos que } \\
\text { ayuden al } \\
\text { desarrollo de } \\
\text { las } \\
\text { neuroactividad } \\
\text { es. }\end{array}$ \\
\hline
\end{tabular}

Tabla 3. Estructura neuromicrocurricular

Los recursos didácticos se integran de forma transversal para lograr la metodología propuesta y no como un componente adicional (Tabla 2).

\section{Conclusiones}

En el cerebro se realizan los procesos de aprendizaje, por ello, este no debe ser aislado de los procesos de enseñanza, ya que la adecuada interpretación de las señales cognitivas ayudará a reinterpretar las cosmovisiones de varias ciencias.

Los estímulos neuronales adecuados son capaces de modificar las conexiones sinápticas del cerebro, favoreciendo el aprendizaje y la memoria. 
En este sentido, los neuromicrocurrículos utilizan las metodologías de enseñanza y aprendizaje para estimular áreas específicas del cerebro y con ello lograr mejores resultados educativos.

Otro eje sobre el cual gira la propuesta para el diseño de neuromicrocurrículos son las emociones, donde se busca estimular de manera preferencial aquellas que den satisfacción al cerebro y al cuerpo, buscando que las emociones positivas creen un anclaje emocional en las áreas cerebrales relacionadas con la memoria a largo plazo.

En resumen, la base de los neuromicrocurrículos se encuentra en el diseño de las metodologías de enseñanza, las cuales están apoyadas por los recursos didácticos apropiados para el desarrollo neuronal de los estudiantes. En pocas palabras, un cerebro feliz, aprende mejor.

\section{References:}

1. Acevedo, S. (2016). Neuromitos en Educación. Abacom, 15(60), 2. Obtenido https://www.centroccbb.cl/abacom/documentos/ABACOM_60.pdf

2. Aguilar, L., Caballero, S., Ormea, V., Salazar, G., Loayza, L., \& Muñoz, A. (2017). La importancia del sueño en el aprendizaje: visos desde la perspectiva de la neurociencia. Avances en psicología, 25(2), 129-137.

3. Barrios, H. (2016). Neurociencias, educación y entorno sociocultural. Educación $\quad y \quad$ Educadores, $19(3), \quad 395-415$. doi:10.5294/edu.2016.19.3.5

4. Caballo, A., \& Portero, M. (2018). 10 ideas claves, Neurociencia y Educación: Aportaciones para el aula. Barcelona: GRAÓ.

5. Campos, A. (2010). Neuroeducación: uniendo las neurociencias y la educación en la búsqueda del desarrollo humano. La Educación(143), $1-14$.

6. Cargua, N. (2005). Currículo I. Obtenido de Universidad Central del Ecuador: http://www.runayupay.org/publicaciones/curriculo_1.pdf

7. Consejo de Educación Superior. (2016). Guía metodológica para la presentación de proyectos de carreras de nivel técnico superior, tecnológico superior y equivalentes. Obtenido de http://www.ces.gob.ec/doc/guia\%20metodologica\%20presentacion\% 20carreras\%20de\%20nive1\%20tyt.pdf

8. De Zubiría, J. (2009). La inteligencia y el talento se desarrollan. Bogotá: Magisterio.

9. Díaz, J., \& Flores, E. (2001). La estructura de la emoción humana: modelo cromático del sistema afectivo. Salud Mental, 24(4), 20-35. 
10. Durant, M., \& Naveda, O. (2012). Transformación curricular por competencia en la educación universitaria bajo un enfoque ecosistemico formativo. Valencia, Venezuela: Ediciones Signos S.

11. Ferreyra, H., Sandrome, D., \& Vidales, S. (2017). Repensar las prácticas de enseñanza en el siglo XXI: planificación, gestión y evaluación. Obtenido de https://www.youtube.com/watch?v=aPN1 nzeiQg

12. Gruart, A. (2014). The role of Neurosciences in Education... and viceversa. International Journal of Educational Psychology, 3(1), 2148.

13. Jung, R. (1992). Some European neuroscientists: a personal tribute. The Neurosciences: Paths of Discovery, 1(1), 477-511.

14. Litwin , E. (2000). Las configuraciones didácticas. Buenos Aires: Paidós SAICF.

15. Martínez, A., \& Petit, K. (2013). Una visión reflexiva sobre la evaluación del microcurrículo y la calidad educativa. Revista Educación en Valores, 1(19), 47-66.

16. Méndez, M., Campi, I., Huayamave, Á., \& Lozada, A. (2017). Modelo de formación dual en el Instituto Tecnológico Superior "Juan Bautista Aguirre". Obtenido de Pontificia Universidad Católica del Ecuador: http://repositorio.pucesa.edu.ec/bitstream/123456789/2309/1/Modali dad.pdf

17. Mendoza, J. (2017). Grado de relación entre expresión emocional y calidad de vida del personal docente y administrativo de las instituciones educativas del distrito de Colcamar, 2016. Tesis de grado. Chachapoyas, Perú: Universidad Nacional Toribio Rodríguez de Mendoza de Amazonas. Obtenido de http://repositorio.untrm.edu.pe/bitstream/handle/UNTRM/1474/MEN DOZA\%20LOPEZ\%20JUAN\%20CARLOS.pdf?sequence=1 \&isAllo wed $=y$

18. Mora, F. (2008). El reloj de la sabiduría. Tiempos y espacios en el cerebro humano. Madrid: Alianza Editorial.

19. Mora, F., \& Sanguinetti, A. (2004). Diccionario de Neurociencias. Madrid: Alianza Editorial.

20. Ocaña, A. (2009). Temas pedagógicos didácticos y metodológicos. Bogotá: Cepedid.

21. Ortiz, A. (2010). Relaciones entre la educación, pedagogía, currículo y didáctica. Dialnet, 6(1), 197-219.

22. Ortiz, A. (2012). Cerebro, currículo y mente humana: Psicología configurante y Pedagogía configuracional. Santa Martha: Edición del autor. 
23. Ortiz, A. (2015). Neuroeducación: ¿Cómo aprende el cerebro humano y cómo deberían enseñar los docentes? Bogotá: Ediciones de la U.

24. Politécnico Superior de Colombia. (2017). Diplomado virtual de neurodidáctica.

Obtenido

de

https://www.politecnicosuperior.com.co/campus/pluginfile.php/1450

63/mod_resource/content/1/GUIA\%20DIDÁCTICA\%201\%20NEUR ODIDACTICA.pdf

25. Rodrigues, J., Abreu, M., \& Castro-Caldas, A. (2013). Neuromyths in education: what is fact and what is fiction for Portuguese teachers? Educational Research, 55(4), 441-453. doi:https://doi.org/10.1080/00131881.2013.844947

26. Tejada, C., \& Tobon, S. (2006). El diseño del plan docente en Información y Documentación acorde con el Espacio Europeo de Educación Superior: un enfoque por competencias. (U. C. Documentación, Ed.) Madrid, España.

27. Urbina, D., \& Agudelo, A. (2016). Análisis de las competencias en formación en comunicación para el desarrollo y cambio social a partir de los micro-currículos del programa de pregrado en comunicación social de la universidad Francisco de Paula Santander, Cúcuta. doi:http://dx.doi.org/10.15332/tg.mae.2016.00475

28. Valdés, H. (2018). Diplomado de Neurodidáctica. Obtenido de Politécnico Superior de Colombia: https://www.politecnicosuperior.com.co/campus/pluginfile.php/1450 64/mod_resource/content/1/NEUROCIENCIAS.pdf

29. Velásquez, B., Remolina, N., \& Calle, M. (2009). El cerebro que aprende. Tabula Rasa, 11, 329-347. 